\section{Auto-avaliação da saúde bucal entre adultos e idosos residentes na Região Sudeste: resultados do Projeto SB-Brasil, 2003}

\author{
Self-rated oral health among Brazilian adults \\ and older adults in Southeast Brazil: \\ results from the SB-Brasil Project, 2003
}

Divane Leite Matos 1,2

Maria Fernanda Lima-Costa 1,2

\footnotetext{
1 Núcleo de Estudos em Saúde Pública e Envelhecimento, Centro de Pesquisas Rene Rachou, Fundação Oswald Cruz/Universidade Federal de Minas Gerais,

Minas Gerais, Brasil.

2 Programa de Pós-graduação em Saúde Pública,

Universidade Federal de Minas Gerais,

Minas Gerais, Brasil.

Correspondência

D. L. Matos

Núcleo de Estudos em Saúde Pública e Envelhecimento, Centro de Pesquisas René

Rachou, Fundação Oswaldo Cruz/Universidade

Federal de Minas Gerais.

Av. Augusto de Lima 1715, sala 609, Belo Horizonte, $M G$ 30190-002, Brasil. divane@cpqrr.fiocruz.br
}

\section{Abstract}

The aim of this study was to determine which characteristics (predisposing and enabling, oral health, perceived need for dental treatment, and behavior) are independently associated with self-rated oral health among adults and older adults in Southeast Brazil. The study was based on 3,240 participants in the SB-Brasil Project/ Southeast. The characteristics of those who rated their oral health as good/very good were compared to those who rated it as fair, poor, or very poor. The following characteristics were significantly and independently associated with better self-rated oral health among adults: monthly household income $\geq U S \$ 60.00$, no current perceived need for dental treatment, place of residence in cities with > 50,000 inhabitants, and visit to the dentist $\geq 3$ years previously. Among older adults the factors were: monthly household income $\geq U S \$ 60.00$, no current perceived need for dental treatment, and 1-19 permanent teeth. Our results confirm those observed in other countries, showing associations between self-rated oral health and predisposing and enabling factors, oral health, perceived need for dental treatment, and behavior.

Oral Health; Adult; Aged

\section{Introdução}

A auto-avaliação da saúde bucal é uma medida que sintetiza a condição objetiva da saúde bucal, a sua funcionalidade e os valores sociais e culturais relacionados à mesma 1,2. Essa avaliação reflete a qualidade de vida, está associada às condições de saúde geral, assim como a comportamentos relacionados aos cuidados com a saúde 1,3 .

Apesar da importância da auto-avaliação da saúde bucal, essa medida ainda é pouco utilizada, principalmente em estudos de base populacional 4. Quase todos os estudos sobre a auto-avaliação da saúde bucal foram realizados em países desenvolvidos, principalmente nos Estados Unidos 1,5,6,7. Estudos sobre a auto-avaliação da saúde bucal são raros no Brasil 2 e, pelo nosso conhecimento, estudos de base populacional sobre o tema são inexistentes neste país.

Entre os estudos conduzidos nos Estados Unidos, um foi realizado entre empregados de duas companhias de seguro 5 e dois foram realizados entre adultos 1 e idosos 6 residentes na cidade de Los Angeles, Califórnia. Um estudo mais abrangente foi conduzido entre pessoas com 35-44 anos e 65-74 residentes em três cidades americanas, que representavam cinco diferentes grupos étnicos 7. A auto-avaliação da saúde bucal como boa ou muito boa predominou de forma consistente em todos os estudos, variando entre 64,2 e $75,9 \%$. Com relação 
aos fatores associados à melhor auto-avaliação da saúde, verificou-se que essa percepção estava associada a perceber melhor a condição geral de saúde 1,6,7, maior número de visitas ao dentista 7 , necessidade auto-referida de tratamento odontológico 1 , presença de maior número de dentes permanentes $1,5,6$, menor número de dentes cariados ou restaurados 1,5, melhor condição periodontal 1,2,5 e indicadores de melhor condição sócio-econômica, tais como maior escolaridade $1,6,7$, maior renda 7 , ou ser branco 1,6 .

No Brasil, a auto-avaliação da saúde bucal foi estudada em 201 idosos (sessenta ou mais anos de idade) atendidos em um centro de saúde na cidade de Araraquara, São Paulo. A maioria dos participantes $(56,4 \%)$ avaliou a sua saúde bucal como excelente a boa e $13 \%$ como ruim ou péssima. A auto-avaliação da saúde bucal apresentou associação independente com três diferentes indicadores da condição de saúde bucal, quais sejam: (a) Geriatric Oral Health Assessment Index (GOHAI) - constituído por 12 perguntas que avaliam se nos últimos três meses o idoso apresentou algum problema funcional, psicológico ou doloroso devido a problemas bucais, (b) presença de dentes com extração indicada e (c) Índice Comunitário de Tratamento e Necessidade Periodontal (CPITN) 2.

Gift et al. 1 elaboraram um modelo explicativo para a auto-avaliação da saúde bucal, incluindo quatro conjuntos de fatores: (a) predisposição e facilitação (recursos que facilitam e provêm meios para o uso de serviços que buscam melhorar ou manter a saúde), (b) nível atual de doenças e condições bucais, (c) percepção da necessidade de tratamento e (d) comportamentos em relação à saúde bucal. No presente trabalho, esse modelo foi utilizado para examinar os fatores associados à auto-avaliação da saúde bucal entre adultos (35-44 anos) e idosos (65-74 anos) residentes na Região Sudeste do Brasil.

\section{Material e métodos}

\section{Fonte de dados}

Foi utilizada a base de dados do Projeto SBBrasil: Condições de Saúde Bucal da População Brasileira. Trata-se de um inquérito realizado pelo Ministério da Saúde/Coordenação Nacional de Saúde Bucal, em parceria com as Secretarias Estaduais e Municipais de Saúde nos anos de 2002 e 2003 8. Esse estudo de base populacional foi realizado em 250 municípios (cinqüenta em cada uma das cinco macrorre- giões brasileiras), considerando seis grupos etários (18-36 meses, 5 anos, 12 anos, 15-19 anos, 35-44 anos e 65-74 anos). A seleção de participantes foi feita por meio de amostra probabilística por conglomerados, obtida em três estágios de seleção: os municípios foram as unidades primárias, os setores censitários foram as unidades secundárias e as quadras e domicílios foram as unidades terciárias de seleção. Todos os moradores dos domicílios selecionados, pertencentes aos grupos etários acima mencionados - e que concordaram em participar foram entrevistados e examinados clinicamente. Na Região Sudeste foram examinados 23.891 indivíduos. Maiores detalhes podem ser vistos em outras publicações 8,9. Para o presente trabalho foram selecionados todos os participantes do Projeto SB-Brasil residentes na Região Sudeste (zona urbana e zona rural), com idades entre 35-44 anos e 65-74 anos.

As entrevistas e exames clínicos foram realizados nos domicílios selecionados. A equipe de campo, em cada cidade, foi constituída por um cirurgião-dentista, responsável pela realização dos exames clínicos, e um auxiliar de consultório dentário ou agente comunitário de saúde, responsável pela realização das entrevistas. Todas as equipes passaram por um processo de treinamento e calibração (intra e interexaminador) objetivando a uniformização dos padrões da entrevista e do exame clínico. A concordância dos exames clínicos intra e interexaminadores foi considerada boa 10 .

Todos os participantes do estudo receberam uma carta explicando os objetivos e procedimentos a serem realizados e assinaram um Termo de Consentimento Livre e Esclarecido. O Projeto SB-Brasil foi aprovado pelo Comitê de Ética em Pesquisa do Ministério da Saúde 8.

\section{Variáveis do estudo}

A variável dependente deste trabalho foi a auto-avaliação da saúde bucal, determinada por meio da seguinte pergunta: "Como classificaria a sua saúde bucal?”, com as respostas variando de "ótima" a "péssima".

A seleção das variáveis independentes deste trabalho foi baseada no modelo explicativo proposto por Gift et al. 1, com pequenas adaptações. As seguintes variáveis de predisposição e facilitação foram consideradas: sexo, número de anos completos de escolaridade, renda domiciliar per capita (agrupada em tercis) e porte do município (até 50 mil habitantes e mais). Para avaliar a condição de saúde bucal foram considerados o número de dentes permanentes presentes e uso de prótese total superior e/ou 
inferior. Necessidade atual autodefinida de tratamento odontológico e o tempo decorrido após a última visita ao dentista foram, respectivamente, as variáveis de necessidade e de comportamento consideradas.

\section{Análise dos dados}

As características daqueles que avaliaram a sua saúde bucal como ótima ou boa foram comparadas às daqueles que avaliaram como regular, ruim ou péssima. A análise foi realizada separadamente para o grupo etário de 35-44 anos e para o grupo etário de 65-74 anos.

$\mathrm{Na}$ análise bivariada utilizou-se o teste do qui-quadrado de Pearson para verificar a existência de associações entre variáveis. O coeficiente de correlação de Pearson foi utilizado para avaliar a existência de colinearidade entre variáveis explicativas. Aquelas variáveis que apresentaram correlação entre fraca a moderada (r até 0,50 ), indicando ausência de colinearidade, foram incluídas nos modelos logísticos.

A análise multivariada foi baseada no método de regressão logística múltipla 11 . Odds ratio (OR) ajustados e seus intervalos de confiança (método de Woolf) em nível de 0,95 (IC95\%) foram utilizadas para estimar as forças das associações entre a auto-avaliação da saúde bucal e as demais variáveis. Foram construídos três modelos logísticos, no primeiro (Modelo 1) foram incluídas todas as variáveis de predisposição e facilitação. No segundo (Modelo 2) foram acrescentadas as variáveis de condição de saúde bucal. No modelo final (Modelo completo), acrescentaram-se as variáveis de necessidade de tratamento e de comportamento, respectivamente. Os dados foram analisados utilizando-se o programa Stata versão 7.0 (Stata Corporation, College Station, Estados Unidos).

\section{Resultados}

Entre os 3.349 participantes com 35-44 e 65-74 anos do inquérito de saúde bucal, na Região Sudeste, $3.240(96,7 \%)$ participaram do presente trabalho; 109 foram excluídos devido à informação incompleta sobre a auto-avaliação da saúde bucal. Entre os participantes, 2.245 e 995 possuíam de 35-44 e 65-74 anos de idade, respectivamente.

Como pode ser visto na Tabela 1 , o sexo feminino predominava entre os participantes (68,1\% dos adultos e $61,4 \%$ dos idosos). Entre os adultos, $84 \%$ possuíam três ou mais anos de escolaridade e $37,8 \%$ tinham renda domiciliar per capita menor ou igual a $\mathrm{R} \$ 80,00$. Entre os
Tabela 1

Distribuição de indicadores das condições de saúde bucal dos participantes do Projeto SB-Brasil, 2003, residentes na Região Sudeste, segundo a faixa etária.

\begin{tabular}{|c|c|c|}
\hline Características & $\begin{array}{l}35-44 \text { anos } \\
(n=2.245) \%\end{array}$ & $\begin{array}{l}65-74 \text { anos } \\
(n=995) \%\end{array}$ \\
\hline \multicolumn{3}{|l|}{ Sexo } \\
\hline Masculino & 31,9 & 38,6 \\
\hline Feminino & 68,1 & 61,4 \\
\hline \multicolumn{3}{|c|}{ Anos completos de escolaridade } \\
\hline Nenhum & 5,4 & 34,8 \\
\hline $1-2$ & 10,6 & 19,1 \\
\hline$\geq 3$ & 84,0 & 46,1 \\
\hline \multicolumn{3}{|c|}{ Renda domiciliar per capita em tercis } \\
\hline 1ㅇ: $\leq \mathrm{R} \$ 80,00$ & 37,8 & 23,8 \\
\hline 2o: $\mathrm{R} \$ 81,00$ a $\mathrm{R} \$ 180,00$ & 32,3 & 36,6 \\
\hline 3ㅇ: $\geq \mathrm{R} \$ 181,00$ & 29,9 & 39,6 \\
\hline \multicolumn{3}{|c|}{ Porte do município de residência (habitantes) } \\
\hline$\leq 50.000$ & 66,5 & 69,4 \\
\hline$>50.000$ & 33,5 & 30,6 \\
\hline \multicolumn{3}{|c|}{ Auto-avaliação da saúde bucal } \\
\hline Ótima & 4,3 & 4,5 \\
\hline Boa & 39,9 & 54,4 \\
\hline Regular & 34,4 & 28,2 \\
\hline Ruim & 13,3 & 8,1 \\
\hline Péssima & 8,1 & 4,8 \\
\hline \multicolumn{3}{|c|}{ Número de dentes permanentes presentes } \\
\hline Nenhum & 11,1 & 65,5 \\
\hline $1-19$ & 27,7 & 25,7 \\
\hline$\geq 20$ & 61,2 & 8,8 \\
\hline \multicolumn{3}{|c|}{ Uso de prótese total superior e/ou inferior } \\
\hline $\operatorname{Sim}$ & 22,1 & 66,3 \\
\hline Não & 77,9 & 33,7 \\
\hline \multicolumn{3}{|c|}{$\begin{array}{l}\text { Necessidade atual autodefinida } \\
\text { de tratamento odontológico }\end{array}$} \\
\hline Sim & 77,0 & 49,8 \\
\hline Não & 23,0 & 50,2 \\
\hline \multicolumn{3}{|c|}{$\begin{array}{l}\text { Tempo decorrido após a última visita } \\
\text { ao dentista (anos) }\end{array}$} \\
\hline$<1$ & 43,0 & 18,8 \\
\hline $1-2$ & 20,8 & 11,6 \\
\hline$\geq 3$ & 36,2 & 69,6 \\
\hline
\end{tabular}

Fonte: Projeto SB-Brasil, Região Sudeste, 2003. 
idosos, as informações correspondentes eram $46,1 \%$ e $23,8 \%$, respectivamente. A maior parte dos participantes, tanto adultos quanto idosos, residia em municípios com população igual ou inferior a 50 mil habitantes $(66,5 \%$ e $69,4 \%$, respectivamente).

No que se refere aos indicadores da saúde bucal (Tabela 1), em ambos os grupos etários, predominou a auto-avaliação da saúde bucal como boa $(39,9 \%$ dos adultos e $54,4 \%$ dos idosos) e regular $(34,4 \%$ e $28,2 \%$, respectivamente). Somente $8,1 \%$ dos adultos e $4,8 \%$ dos idosos avaliaram a saúde bucal como péssima. Entre os primeiros, $11 \%$ não possuíam dentes naturais, sendo essa proporção igual a $65,5 \%$ entre os idosos. O uso de prótese total superior e/ ou inferior foi igual a $22 \%$ e $66 \%$, respectivamente. Entre os adultos, $43 \%$ haviam visitado o dentista há menos de um ano, ao passo que somente $19 \%$ dos idosos haviam visitado o dentista nesse período.

Na Tabela 2 estão apresentados os resultados da análise bivariada da auto-avaliação da saúde bucal e sua associação com características selecionadas, segundo a faixa etária. Todas as variáveis consideradas apresentaram associações significantes $(p<0,05)$ com auto-avaliação da saúde bucal, com exceção de sexo ( $\mathrm{p}=$ 0,690 ) e anos completos de escolaridade $(0,236)$ para os adultos e porte do município $(\mathrm{p}=0,679)$ e anos completos de escolaridade $(0,449)$ para os idosos.

Em ambos os grupos etários, todos os coeficientes de correlação de Pearson entre as variáveis independentes deste trabalho foram inferiores a 0,50, com exceção para o uso de prótese total superior e/ou inferior com o número de dentes permanentes, no grupo etário 35-44 anos, cujo valor de $\mathrm{r}$ foi igual a $-0,74$ (Tabela 3 ).

Na Tabela 4 estão apresentados os resultados da análise multivariada das características associadas à auto-avaliação da saúde bucal como ótima/boa entre os adultos (35-44 anos). No modelo final completo, renda domiciliar per capita maior ou igual a $\mathrm{R} \$ 181,00(\mathrm{OR}=1,54$; IC95\%: 1,23-1,94) e percepção de não necessidade atual de tratamento odontológico (OR = 4,10; IC95\%: 3,26-5,17) permaneceram positiva e significativamente associadas à melhor autoavaliação da saúde bucal. Associações significantes e negativas foram encontradas para município com mais de 50 mil habitantes (OR = 0,58; IC95\%: 0,48-0,71) e três ou mais anos decorridos após a última visita ao dentista $(\mathrm{OR}=$ 0,67; IC95\%: 0,54-0,83).

Os resultados da análise multivariada das características associadas à auto-avaliação da saúde bucal como ótima/boa entre os idosos
(65-74 anos) estão apresentados na Tabela 5. No modelo completo, associações significantes e positivas com melhor auto-avaliação da saúde bucal foram observadas para: renda domiciliar per capita maior ou igual a $\mathrm{R} \$ 181,00(\mathrm{OR}=$ 1,99; IC95\%: 1,39-2,86) e percepção de não necessidade atual de tratamento odontológico ( $\mathrm{OR}=3,27$; IC95\%: 2,45-4,35). Associação significante e negativa foi observada para presença de 1 a 19 dentes (OR = 0,60; IC95\%: 0,42-0,85).

\section{Discussão}

A auto-avaliação da saúde bucal como boa e ótima (59\%) predominou entre os idosos participantes deste estudo. Essa proporção entre os mais jovens foi menor (44\%). A prevalência da auto-avaliação como boa e ótima entre os idosos foi inferior ao observado entre participantes de um estudo de base populacional realizado em Los Angeles (76\%) 6, mas semelhante ao observado entre clientes idosos do Centro de Saúde de Araraquara, em São Paulo 2. A prevalência de adultos que auto-avaliavam a sua saúde como boa e ótima no presente trabalho foi inferior ao observado em inquéritos norteamericanos 1,5 .

Chama a atenção neste trabalho a melhor auto-avaliação da saúde bucal entre os idosos do que entre os mais jovens. Em um estudo desenvolvido entre idosos norte-americanos residentes na comunidade, verificou-se que cerca de $40 \%$ daqueles que haviam perdido mais da metade dos seus dentes avaliavam sua saúde bucal como excelente ou boa 6 . Em outros trabalhos 3,6,7 verificou-se que os idosos aceitavam a perda de dentes mais facilmente, por considerarem que essas perdas eram resultantes de um processo natural do envelhecimento. Com isto, a condição de saúde bucal era superestimada. Além disso, verifica-se que alguns idosos, devido a repetidos problemas com seus dentes naturais, consideram haver uma real melhora da saúde bucal com a substituição dos mesmos por próteses parciais ou totais. Segundo Silva \& Fernandes 2 , o fato de as principais doenças bucais apresentarem caráter não letal, leva ao aceite das mesmas como inevitáveis (“conformismo"). Essa aceitação é reforçada pelo fato de os idosos pertencerem a uma coorte na qual, além da alta prevalência de cárie, os tratamentos eram baseados na extração e na colocação de próteses totais, independente da classe social. Isto pode explicar a melhor autoavaliação da saúde bucal observada entre os idosos, em comparação aos mais jovens, observada neste e em outros estudos 3,5,6,12 . 
Análise bivariada da auto-avaliação da saúde bucal como ótima ou boa e sua associação com características selecionadas entre participantes do Projeto SB-Brasil, 2003, residentes na Região Sudeste, segundo a faixa etária.

\begin{tabular}{|c|c|c|c|c|c|c|}
\hline \multirow[t]{4}{*}{ Características } & \multicolumn{6}{|c|}{ Auto-avaliação da saúde bucal como ótima/boa } \\
\hline & \multicolumn{3}{|c|}{ 35-44 anos } & \multicolumn{3}{|c|}{ 65-74 anos } \\
\hline & $\begin{array}{c}\operatorname{Sim}_{(n=992} \\
(n=10\end{array}$ & $\begin{array}{l}\text { Não } \\
=1.253)\end{array}$ & $\mathrm{p}$ & $\begin{array}{c}\operatorname{Sim}_{(n=586)} \\
(n=5)\end{array}$ & $\begin{array}{c}\text { Não } \\
(n=409)\end{array}$ & p \\
\hline & & $\%$ & & & $\%$ & \\
\hline \multicolumn{7}{|l|}{ Predisposição e facilitação } \\
\hline \multicolumn{7}{|l|}{ Sexo } \\
\hline Masculino & 31,5 & 32,2 & & 35,8 & 42,5 & \\
\hline Feminino & 68,5 & 67,8 & 0,690 & 64,2 & 57,5 & 0,033 \\
\hline \multicolumn{7}{|c|}{ Anos completos de escolaridade } \\
\hline Nenhum & 4,8 & 5,9 & & 36,4 & 32,5 & \\
\hline $1-2$ & 9,7 & 11,3 & & 18,4 & 20,1 & \\
\hline$\geq 3$ & 85,5 & 82,8 & 0,236 & 45,2 & 47,4 & 0,449 \\
\hline \multicolumn{7}{|c|}{ Renda domiciliar per capita em tercis } \\
\hline 1 ㅇ: $\leq \mathrm{R} \$ 80,00$ & 33,3 & 41,4 & & 19,1 & 30,6 & \\
\hline 2o: $R \$ 81,00$ a $R \$ 180,00$ & 31,6 & 33,0 & & 36,9 & 36,2 & \\
\hline 3ㅇ: $\geq R \$ 181,00$ & 35,2 & 25,6 & 0,000 & 44,0 & 33,2 & 0,000 \\
\hline \multicolumn{7}{|c|}{ Porte do município de residência (habitantes) } \\
\hline$\leq 50.000$ & 71,5 & 62,6 & & 68,9 & 70,2 & \\
\hline$>50.000$ & 28,5 & 37,4 & 0,000 & 31,1 & 29,8 & 0,679 \\
\hline \multicolumn{7}{|l|}{ Condição de saúde bucal } \\
\hline \multicolumn{7}{|c|}{ Número de dentes permanentes presentes } \\
\hline Nenhum & 14,2 & 8,6 & & 73,0 & 54,8 & \\
\hline $1-19$ & 24,1 & 30,6 & & 19,8 & 34,2 & \\
\hline$\geq 20$ & 61,7 & 60,8 & 0,000 & 7,2 & 11,0 & 0,000 \\
\hline \multicolumn{7}{|c|}{ Uso de prótese total superior e/ou inferior } \\
\hline Sim & 25,5 & 19,3 & & 69,3 & 62,1 & \\
\hline Não & 74,5 & 80,7 & 0,001 & 30,7 & 37,9 & 0,018 \\
\hline \multicolumn{7}{|c|}{ Necessidade de tratamento e comportamento } \\
\hline \multicolumn{7}{|c|}{$\begin{array}{l}\text { Necessidade atual autodefinida } \\
\text { de tratamento odontológico }\end{array}$} \\
\hline Sim & 63,2 & 88,0 & & 36,2 & 69,4 & \\
\hline Não & 36,8 & 12,0 & 0,000 & 63,8 & 30,6 & 0,000 \\
\hline \multicolumn{7}{|c|}{$\begin{array}{l}\text { Tempo decorrido após a última visita } \\
\text { ao dentista (anos) }\end{array}$} \\
\hline$<1$ & 48,0 & 39,0 & & 16,4 & 22,2 & \\
\hline $1-2$ & 21,6 & 20,1 & & 10,6 & 13,2 & \\
\hline$\geq 3$ & 30,4 & 40,9 & 0,000 & 73,0 & 64,6 & 0,020 \\
\hline
\end{tabular}

Fonte: Projeto SB-Brasil, Região Sudeste, 2003. 
Tabela 3

Coeficiente de correlação de Pearson entre variáveis explicativas do estudo em participantes do Projeto SB-Brasil, 2003, residentes na Região Sudeste, segundo a faixa etária.

\begin{tabular}{|c|c|c|c|c|c|c|c|c|}
\hline \multicolumn{2}{|c|}{ Variáveis } & \multicolumn{7}{|c|}{ Variáveis } \\
\hline & Grupo etário de $35-44$ anos & 1 & 2 & 3 & 4 & 5 & 6 & 7 \\
\hline 1 & Sexo* & - & & & & & & \\
\hline 2 & Anos completos de escolaridade ${ }^{\star \star}$ & 0,02 & - & & & & & \\
\hline 3 & Renda domiciliar per capita em tercis ${ }^{\star \star \star}$ & $-0,04$ & 0,39 & - & & & & \\
\hline 4 & Porte do município de residência\# & $-0,02$ & 0,08 & 0,14 & - & & & \\
\hline 5 & Número de dentes permanentes\#\# & $-0,10$ & 0,25 & 0,19 & 0,07 & - & & \\
\hline 6 & Uso de prótese total superior e/ou inferior\#\#\# & 0,14 & $-0,17$ & $-0,13$ & $-0,05$ & $-0,74$ & - & \\
\hline 7 & Necessidade atual autodefinida de tratamento odontológico§ & $-0,00$ & 0,05 & 0,09 & 0,03 & $-0,19$ & 0,19 & - \\
\hline \multirow[t]{2}{*}{8} & Tempo decorrido após a última visita ao dentista§§ & $-0,05$ & $-0,26$ & $-0,19$ & 0,02 & $-0,19$ & 0,17 & $-0,06$ \\
\hline & Grupo etário de $65-74$ anos & & & & & & & \\
\hline 1 & Sexo* & - & & & & & & \\
\hline 2 & Anos completos de escolaridade ${ }^{\star \star}$ & $-0,06$ & - & & & & & \\
\hline 3 & Renda domiciliar per capita em tercis ${ }^{\star \star *}$ & $-0,01$ & 0,16 & - & & & & \\
\hline 4 & Porte do município de residência\# & $-0,00$ & 0,17 & 0,11 & - & & & \\
\hline 5 & Número de dentes permanentes\#\# & $-0,16$ & 0,15 & 0,07 & 0,03 & - & & \\
\hline 6 & Uso de prótese total superior e/ou inferior\#\#\# & 0,16 & 0,05 & 0,01 & $-0,02$ & $-0,49$ & - & \\
\hline 7 & Necessidade atual autodefinida de tratamento odontológico§ & 0,08 & $-0,07$ & 0,07 & 0,00 & $-0,25$ & 0,11 & - \\
\hline 8 & Tempo decorrido após a última visita ao dentista§§ & 0,00 & $-0,35$ & $-0,05$ & $-0,08$ & $-0,30$ & 0,08 & 0,12 \\
\hline
\end{tabular}

* Sexo $=1$ : masculino, 2 : feminino;

** Anos completos de escolaridade $=1$ : nenhum, 2: 1-2, 3: $\geq 3$

$\star \star \star$ Renda domiciliar per capita em tercis $=1$ ㅇ: $\leq \mathrm{R} \$ 80,00,2$ ㅇ: $\mathrm{R} \$ 81,00$ a $\mathrm{R} \$ 180,00$ e 3 o: $\geq \mathrm{R} \$ 181,00$.

\# Porte do município de residência (habitantes) = 1: $\leq 50.000$ e 2: > 50.000;

\#\# Número de dentes permanentes $=1$ : nenhum, 2: 1 a 19 e $3: \geq 20$;

\#\#\# Uso de prótese total superior e/ou inferior = 1: $\operatorname{sim}$ e 2: não;

$\S$ Necessidade atual autodefinida de tratamento odontológico $=1$ : $\operatorname{sim}$ e 2: não:

$\S \S$ Tempo decorrido após a última visita ao dentista (anos) $=1:<1,2: 1-2$ e $3: \geq 3$.

De uma maneira geral, observa-se que a boa saúde bucal percebida é o mais importante preditor da percepção da não necessidade atual de tratamento odontológico. Nossos resultados confirmam essas observações. No presente trabalho, a renda domiciliar per capita, mas não a escolaridade, ocupou uma posição central na predição da melhor auto-avaliação da saúde bucal, tanto entre adultos quanto entre idosos. Estudos realizados nos Estados Unidos e na Nova Zelândia 1,3,5 verificaram que em ambos os grupos etários a classe social e/ou a escolaridade não estavam associadas à auto-avaliação da saúde bucal. O estudo neozelandês 3 mostrou que a não associação entre fatores sócio-econômicos e auto-avaliação da saúde bucal entre adultos daquele país pode ser explicada pelo fato de que os serviços de saúde têm um efeito igualitário, anulando o impacto desses fatores.

Para os adultos, o fato de ter visitado o dentista há três ou mais anos foi importante para aumentar as chances de auto-avaliar a saúde bucal como ruim. Entre os idosos, não foi encontrada associação significativa entre tempo decorrido após a última visita ao dentista e auto-avaliação da saúde bucal. É possível que alta prevalência de edentulismo entre idosos (66\% não possuíam dentes naturais) torna, na sua percepção, desnecessária a visita ao dentista.

Entre idosos, a melhor percepção da saúde bucal esteve associada ao número de dentes presentes. Ao contrário do esperado, aqueles que possuíam entre 1 e 19 dentes avaliaram a sua saúde bucal como pior, em comparação com aqueles que não possuíam dentes. Talvez este fato pode ser explicado pela qualidade dos dentes remanescentes, gerando dor ou insatisfação com a mastigação e estética, e também devido ao grande número de dentes naturais perdidos. Como demonstrado em muitos estudos 5,6, o número de dentes perdidos tem uma grande influência na auto-avaliação da saúde bucal, principalmente entre idosos.

O presente trabalho possui os limites impostos pelo uso de dados secundários. As duas 
Resultados da análise multivariada dos fatores associados à auto-avaliação da saúde bucal como ótima ou boa entre participantes do Projeto SB-Brasil, 2003, residentes na Região Sudeste, com idade entre 35-44 anos.

\begin{tabular}{|c|c|c|c|}
\hline & \multicolumn{3}{|c|}{$\begin{array}{c}\text { Auto-avaliação da condição de saúde } \\
\text { bucal como ótima/boa* }\end{array}$} \\
\hline & $\begin{array}{l}\text { Modelo } 1 \\
\text { OR ajustado } \\
(\text { IC } 95 \%)^{\star \star}\end{array}$ & $\begin{array}{l}\text { Modelo } 2 \\
\text { OR ajustado } \\
(\text { IC95\%)**}\end{array}$ & $\begin{array}{c}\text { Modelo completo } \\
\text { OR ajustado } \\
(\text { IC } 95 \%)^{\star *}\end{array}$ \\
\hline \multicolumn{4}{|l|}{ Predisposição e facilitação } \\
\hline \multicolumn{4}{|c|}{ Renda domiciliar per capita em tercis } \\
\hline 1 ㅇ: $\leq \mathrm{R} \$ 80,00$ & 1,00 & 1,00 & 1,00 \\
\hline 2o: $\mathrm{R} \$ 81,00$ a $\mathrm{R} \$ 180,00$ & $1,24(1,01-1,52)$ & $1,25(1,02-1,54)$ & $1,15(0,93-1,43)$ \\
\hline 3ㅇ: $\geq \mathrm{R} \$ 181,00$ & $1,86(1,51-2,29)$ & $1,91(1,54-2,36)$ & $1,54(1,23-1,94)$ \\
\hline \multicolumn{4}{|c|}{ Porte do município de residência (habitantes) } \\
\hline$\leq 50.000$ & 1,00 & 1,00 & 1,00 \\
\hline$>50.000$ & $0,62(0,51-0,74)$ & $0,62(0,52-0,75)$ & $0,58(0,48-0,71)$ \\
\hline \multicolumn{4}{|l|}{ Condição de saúde bucal } \\
\hline \multicolumn{4}{|c|}{ Número de dentes permanentes presentes } \\
\hline Nenhum & & 1,00 & 1,00 \\
\hline $1-19$ & & $0,47(0,35-0,64)$ & $0,72(0,52-1,01)$ \\
\hline$\geq 20$ & & $0,56(0,43-0,74)$ & $0,86(0,63-1,17)$ \\
\hline \multicolumn{4}{|c|}{ Necessidade de tratamento e comportamento } \\
\hline \multicolumn{4}{|c|}{$\begin{array}{l}\text { Necessidade atual autodefinida de tratamento } \\
\text { odontológico }\end{array}$} \\
\hline Sim & & & 1,00 \\
\hline Não & & & $4,10(3,26-5,17)$ \\
\hline \multicolumn{4}{|c|}{ Tempo após a última visita ao dentista (anos) } \\
\hline$<1$ & & & 1,00 \\
\hline $1-2$ & & & $0,94(0,74-1,19)$ \\
\hline$\geq 3$ & & & $0,67(0,54-0,83)$ \\
\hline
\end{tabular}

Fonte: Projeto SB-Brasil, Região Sudeste, 2003.

* A classe de referência foi a auto-avaliação da saúde bucal como regular/ruim/péssima;

** OR ajustado (IC95\%), ajustados por todas as variáveis listadas na tabela, usando regressão

logística múltipla (2.193 indivíduos participaram do modelo final).

principais limitações referem-se ao delineamento seccional do Projeto SB-Brasil e a participação no estudo. O delineamento seccional é adequado para estimar a prevalência da autoavaliação da saúde bucal na população estudada, mas ele não permite determinar se existe relação temporal entre esta e as variáveis independentes consideradas neste trabalho. Além disso, existe alguma evidência de que a seleção dos participantes atuou no sentido de aumentar a proporção de pessoas do sexo feminino. Por exemplo, o Censo Brasileiro de 2000 mostra que nas faixas etárias de 35-44 anos e de 65-74 anos de idade, a proporção de mulheres na Região Sudeste é igual a 51,4\% e 55,1\%, respectivamente. As proporções correspondentes de participantes no Projeto SB-Brasil são iguais a
$68,1 \%$ e $61,4 \%$. Embora isso possa ter comprometido a validade externa deste estudo, não existem evidências de ter comprometido a validade interna. Por outro lado, o Projeto SBBrasil é o primeiro grande inquérito de saúde bucal neste país que produziu informações abrangentes sobre a saúde bucal da população idosa, incluindo exame clínico. Os inquéritos anteriores consideravam somente crianças em idade escolar e, somente um incluiu pessoas com idade igual ou inferior a 59 anos 13. O suplemento de saúde da Pesquisa Nacional por Amostra de Domicílios incluiu algumas informações sobre a saúde bucal das populações adulta e idosa brasileiras, mas essas informações são restritas ao uso de serviços de saúde bucal 14. Desta forma, as informações do Proje- 
Resultados da análise multivariada dos fatores associados à auto-avaliação da saúde bucal como ótima ou boa entre participantes do Projeto SB-Brasil, 2003, residentes na Região Sudeste, com idade entre 65-74 anos.

\begin{tabular}{|c|c|c|c|}
\hline & \multicolumn{3}{|c|}{$\begin{array}{c}\text { Auto-avaliação da condição de saúde } \\
\text { bucal como ótima/boa* }\end{array}$} \\
\hline & $\begin{array}{l}\text { Modelo } 1 \\
\text { OR ajustado } \\
(\text { IC95\%)** }\end{array}$ & $\begin{array}{l}\text { Modelo } 2 \\
\text { OR ajustado } \\
(\text { IC } 95 \%)^{\star \star}\end{array}$ & $\begin{array}{c}\text { Modelo completo } \\
\text { OR ajustado } \\
(\text { IC } 95 \%)^{\star *}\end{array}$ \\
\hline \multicolumn{4}{|l|}{ Predisposição e facilitação } \\
\hline \multicolumn{4}{|l|}{ Sexo } \\
\hline Masculino & 1,00 & 1,00 & 1,00 \\
\hline Feminino & $1,34(1,03-1,74)$ & $1,17(0,89-1,53)$ & $1,20(0,90-1,60)$ \\
\hline \multicolumn{4}{|c|}{ Renda domiciliar per capita em tercis } \\
\hline 1ㅇ: $\leq \mathrm{R} \$ 80,00$ & 1,00 & 1,00 & 1,00 \\
\hline 2o: $\mathrm{R} \$ 81,00$ a $\mathrm{R} \$ 180,00$ & $1,65(1,18-2,29)$ & $1,59(1,13-2,23)$ & $1,40(0,98-2,01)$ \\
\hline 3ㅇ: $\geq \mathrm{R} \$ 181,00$ & $2,13(1,53-2,97)$ & $2,28(1,62-319)$ & $1,99(1,39-2,86)$ \\
\hline \multicolumn{4}{|l|}{ Condição de saúde bucal } \\
\hline \multicolumn{4}{|c|}{ Número de dentes permanentes presentes } \\
\hline Nenhum & & 1,00 & 1,00 \\
\hline $1-19$ & & $0,42(0,31-0,58)$ & $0,60(0,42-0,85)$ \\
\hline$\geq 20$ & & $0,44(0,26-0,74)$ & $0,64(0,35-1,15)$ \\
\hline \multicolumn{4}{|c|}{ Uso de prótese total superior e/ou inferior } \\
\hline Sim & & 1,00 & 1,00 \\
\hline Não & & $0,93(0,67-1,29)$ & $0,87(0,62-1,24)$ \\
\hline \multicolumn{4}{|c|}{ Necessidade de tratamento e comportamento } \\
\hline \multicolumn{4}{|c|}{$\begin{array}{l}\text { Necessidade atual autodefinida de tratamento } \\
\text { odontológico }\end{array}$} \\
\hline Sim & & & 1,00 \\
\hline Não & & & $3,27(2,45-4,35)$ \\
\hline \multicolumn{4}{|c|}{ Tempo após a última visita ao dentista (anos) } \\
\hline$<1$ & & & 1,00 \\
\hline $1-2$ & & & $1,15(0,69-1,90)$ \\
\hline$\geq 3$ & & & $1,22(0,85-1,77)$ \\
\hline
\end{tabular}

Fonte: Projeto SB-Brasil, Região Sudeste, 2003.

* A classe de referência foi a auto-avaliação da saúde bucal como regular/ruim/péssima;

** OR ajustado (IC95\%), ajustados por todas as variáveis listadas na tabela, usando regressão logística múltipla (965 indivíduos participaram do modelo final).

to SB-Brasil permitiram, pela primeira vez neste país, o estudo dos fatores associados à autoavaliação da saúde bucal em uma grande base populacional de adultos e idosos.

Finalizando, os resultados deste trabalho mostram que a auto-avaliação da saúde bucal em adultos e idosos é explicada por variáveis de predisposição, facilitação, além da necessi- dade de tratamento e comportamento. Esses resultados mostram uma estrutura multidimensional da auto-avaliação da saúde bucal, indicando que o modelo explicativo proposto Gift et al. 1, pode ser aplicado em populações diferentes daquelas nas quais ele foi originalmente concebido. 


\section{Resumo}

O objetivo deste estudo foi verificar quais fatores de predisposição e facilitação, da condição de saúde bucal, de necessidade de tratamento e de comportamento estão associados à auto-avaliação da saúde bucal entre adultos (35-44 anos) e idosos (65-74 anos) residentes na Região Sudeste do Brasil. Fizeram parte deste trabalho 3.240 pessoas participantes do Projeto $S B$ Brasil/Região Sudeste. As características daqueles que avaliaram a sua saúde bucal como ótima ou boa foram comparadas às daqueles que avaliaram como regular, ruim ou péssima. No modelo final, as características independentemente associadas à melhor autoavaliação da saúde bucal entre os adultos foram renda domiciliar per capita $\geq R \$ 181,00$, não necessidade atual de tratamento odontológico, município de residência com mais de 50 mil habitantes e visita ao dentista há $\geq 3$ anos. Entre os idosos foram: renda domiciliar per capita $\geq R \$ 181,00$, não necessidade atual de tratamento odontológico e possuir entre 1 a 19 dentes. Nossos resultados confirmam estudos de outros países, mostrando a existência de associações entre auto-avaliação e fatores de predisposição e facilitação, condição de saúde bucal, necessidade de tratamento e comportamento.

Saúde Bucal; Adulto; Idoso

\section{Colaboradores}

D. L. Matos foi responsável pela análise dos dados e redação do trabalho. M. F. Lima-Costa foi orientadora do trabalho, tendo sido responsável pela supervisão da análise dos dados, redação do trabalho e revisão final do artigo.

\section{Agradecimentos}

À Coordenação Nacional de Saúde Bucal do Ministério da Saúde pela cessão do banco de dados e aos examinadores e anotadores responsáveis pela coleta dos dados. Sem a adesão da população dos municípios selecionados este estudo seria inviável.

\section{Referências}

1. Gift HC, Atchison KA, Drury TF. Perceptions of the natural dentition in the context of multiple variables. J Dent Res 1998; 77:1529-38.

2. Silva SRC, Fernandes RAC. Autopercepção das condições de saúde bucal por idosos. Rev Saúde Pública 2001; 35:349-55.

3. Chen MS, Hunter P. Oral health and quality of life in New Zealand: a social perspective. Soc Sci Med 1996; 43:1213-22.

4. Atchison KA, Matthias RE, Dolan TA, Lubben JE, De Jong F, Mayer-Oakes SA. Comparison of oral health ratying by dentists and dentate elders. J Public Health Dent 1993, 53:223-30.

5. Reisine ST, Bailit HL. Clinical oral health status and adult perceptions of oral health. Soc Sci Med 1980; 14:597-605.

6. Matthias RE, Atchison KA, Lubben JE, De Jong F, Schweitzer SO. Factors affeting self-ratings of oral health. J Public Health Dent 1995; 55:197-204.

7. Atchison KA, Gift HC. Perceived oral health in a diverse sample. Adv Dent Res 1997; 11:272-80.

8. Roncalli AG, Frazão P, Patussi MP, Araújo IC, Ely HC, Batista SM. Projeto SB2000: uma perspectiva para a consolidação da Epidemiologia em Saúde Bucal Coletiva. Rev Bras Odont Saúde Coletiva 2000; 1:9-25.

9. Ministério da Saúde. Projeto SB2000: condições de saúde bucal da população brasileira no ano de 2000. Manual do coordenador. Brasília: Ministério da Saúde; 2001.

10. Ministério da Saúde. Projeto SB2000: condições de saúde bucal da população brasileira no ano de 2000. Manual de calibração dos examinadores. Brasília: Ministério da Saúde; 2001.

11. Hosmer DW, Lemeshow S. Applied logistic regression. New York: Johns Wiley \& Sons; 1989.

12. Silva DD, Sousa MLR, Wada RS. Autopercepção e condições de saúde bucal em uma população de idosos. Cad Saúde Pública 2005; 21:1251-9.

13. Oliveira AGRC. Perfil epidemiológico de saúde bucal no Brasil 1986-1996. http://www.angelonline.cjb.net (acessado em 28/Jul/2005).

14. Matos DL, Giatti L, Lima-Costa MF. Fatores sóciodemográficos associados ao uso de serviços odontológicos entre idosos brasileiros: um estudo baseado na Pesquisa Nacional por Amostra de Domicílios. Cad Saúde Pública 2004; 20:1290-7.

Recebido em 31/Ago/2005

Versão final reapresentada em 02/Dez/2005

Aprovado em 06/Dez/2005 